\title{
Externally driven local colloidal ordering induced by a pointlike heat source
}

\author{
Nicolas Bruot $\odot^{1,2, *}$ and Hajime Tanaka $\odot^{1, \dagger}$ \\ ${ }^{1}$ Institute of Industrial Science, University of Tokyo, 4-6-1 Komaba, 153-8505 Meguro-ku, Tokyo, Japan \\ ${ }^{2}$ Institut de Physique de Nice, CNRS, UMR No. 7010, Université Côte d'Azur, 28 Avenue Joseph Vallot, 06108 Nice, France
}

(Received 30 July 2019; published 24 December 2019)

\begin{abstract}
We study here how phase transitions are induced in colloidal suspensions by a pointlike heat source, an optically trapped metal-oxide-particle absorbing light. We find that thermophoresis increases the number density of colloids around the oxide particle, leading to the appearance of solid clusters. Our analysis based on thermophoresis reveals that the solid-fluid interface position is purely determined by the relationship of the particle concentration profile in the fluid state with the volume fraction of the phase transition, and no other effect of thermodynamics is seen in the cluster sizes. In this system, we observe the formation of face-centered-cubic crystals, amorphous states, and structures with icosahedral order. This shows a rich possibility of nontrivial orderings under spatially controlled heterogeneous growth in external semisoft potentials that are softer than walls but with substantial variations at the scale of a few particle diameters. Because of the tunable rate of the addition of particles to the clusters, we propose that this method could be used to study the complex interplay of densification with local spatial confinement effects, kinetics, and ordering.
\end{abstract}

DOI: 10.1103/PhysRevResearch.1.033200

\section{INTRODUCTION}

Colloidal particles dispersed in a solvent are excellent systems to investigate phase transition phenomena as the particles experience thermal fluctuations and at the same time they are big enough to be observed at the particle-level resolution with common microscopy techniques and at relevant timescales [1]. In particular, confocal microscopy observations provide microscopic information like individual particle locations and dynamics, which has tremendously contributed to the fundamental understanding of equilibrium and nonequilibrium phase transitions. They include various phenomena such as crystal nucleation [2,3], glass transition [4-11], phase separation, and gelation [12-15].

Since phase transitions of colloids can be affected by external fields, we may induce various types of transitions by applying external fields in a reversible fashion, such as optical [16-18], magnetic [19-21], electric [20-22], or thermal fields (taking advantage of either thermophoresis [23-25] or temperature-dependant particle size [26,27]). Among these, the optical field is particularly interesting since it can be spatially localized in a region smaller than the size of colloids. In particular, local heating due to light absorption provides a convenient way to induce localized phase transitions under open out-of-equilibrium conditions [28,29]. Local heating may perturb colloidal suspensions in a variety of ways. It

\footnotetext{
"nicolas@ bruot.org

†tanaka@iis.u-tokyo.ac.jp
}

Published by the American Physical Society under the terms of the Creative Commons Attribution 4.0 International license. Further distribution of this work must maintain attribution to the author(s) and the published article's title, journal citation, and DOI. should lead to more vigorous Brownian motion in the heated region. Furthermore, a coupling to colloidal particles with a temperature gradient, termed the thermophoretic effect, leads to migration or depletion, i.e., the local change in the volume fraction of colloids $\phi$ in a heated region $[28,30]$. Local heating may also induce convective flows if it is strong enough [31]. Since many colloid phase transitions are induced by the change in $\phi$, we may induce phase transitions by spatially modulating $\phi$ via such a coupling. It opens up a new type of localized phase transitions under energy input, applicable to gas-liquid transitions, crystallization, glass transition, phase separation, and gelation. For example, Jiang et al. [24] found that migration of colloids can be driven by a nonuniform polymer distribution sustained by the polymer's thermophoresis due to laser heating. These studies may serve as model cases of phase transitions taking place in nature under a variety of inhomogeneous fields in a complex manner.

In this article, we study local heating effects on phase transitions and packing of the simplest possible colloidal system commonly used to study phase transitions: hard spheres. We create a very localized inhomogeneous temperature field by shining a laser on a titanium dioxide particle immersed in a colloidal suspension. This oxide sphere (impurity) is both trapped and heated by optical tweezers, inducing strong thermophoretic effects, which move the particles of the suspension towards the hot oxide particle. The direction of particle motion is opposite to that expected for uncharged particles [32], indicating crucial roles of surface charges and/or chemistry, and solvent properties. As a consequence of inhomogeneous densification, we observe unconventional nucleation and growth of condensed solid phases and other states (crystals, structures with icosahedral order, and amorphous solids) under the inhomogeneous temperature field. The steady-state size and dynamics of growth are investigated. 


\section{MATERIALS AND METHODS}

The samples are nearly the same as those described in Refs. [14,15] and we only describe here their main features and the novelties. Poly(methyl methacrylate) (PMMA) colloids stabilized with metacryloxypropyl-terminated poly(dimethylsiloxane) and containing a small amount of rhodamine-B dye were synthesized. We always used organic solvents that were kept dry by storing them in contact with molecular sieves (Sigma-Aldrich, rods, 3A, 1/8). We suspended the spherical colloids of radii varying from $a=0.15$ to $1.2 \mu \mathrm{m}$ in a solution of about $20 \%$ of cisdecahydronaphtalene (cis-decalin, $>98 \%, \quad$ TCI) and bromocyclohexane $(98 \%$, Aldrich) saturated in tetra- $n$ butylammonium bromide salt (Wako). Such samples are known to have interactions and phase behavior close to hard spheres [9,13-15]. Samples with different volume fractions between $5 \%$ and $30 \%$, far from the freezing point ( 49.4\%), were prepared. In these mixtures, the particles were refractive-index matched and the exact ratio of the solvents was tuned to have a good density match that prevented the sedimentation of the particles for $10 \mathrm{~h}$ at least. Separately, titania metal-oxide particles of radius $R_{0}=1.5 \mu \mathrm{m}$, which were initially in an aqueous solution (Corpuscular, $-\mathrm{COOH}$ functionalized), were transferred to cis-decalin using ethanol (99.5\%, Wako) as an intermediate solvent. Then $2 \mu \mathrm{l}$ of this solution was added to $200 \mu 1$ of the solution of PMMA particles and stirred. Such solutions of PMMA colloids and titania particles were sealed in glass capillary tubes suitable for optical trapping and confocal microscopy observation (VitroTubes, $100 \mu \mathrm{m} \times 2 \mathrm{~mm} \times 50 \mathrm{~mm}$ ). We found that it was necessary to coat the walls of the samples to prevent the titania particles from sticking to the glass. Therefore, the inside of the capillaries with two parallel walls spaced by $100 \mu \mathrm{m}$ was covered with a thin layer of PMMA by flowing a solution of PMMA dissolved in toluene and then flowing nitrogen to spread the solution on the inner glass surfaces and to remove the excess solution and evaporate the toluene. This resulted in a thin solid PMMA film of thickness $\lesssim 1 \mu \mathrm{m}$ on all the inner surfaces of the sample, which was checked by confocal microscopy in test samples using a fluorescent PMMA/toluene solution. The capillaries were finally filled with a solution of PMMA colloids and titania particles and then put on a microscope slide and sealed with UV-cured glue (NOA 68). The samples were not filled entirely so that air initially separated the glue and the solution. The presence of air provided three advantages. First, the liquid glue was never in contact with the solution, which limited the possibility of compounds from the glue being dissolved in the solution before it was cured. Second, the center of the sample containing the solution could easily be protected from UV light during curing, which was necessary as we found that long direct UV illumination led to particles sticking to each other and forming large solid three-dimensional structures. Third, the solution in the finished capillaries could be stirred by shaking the sample vigorously to make air bubbles travel several times from one side of the sample to the other. Such shaking was done at the beginning of each day of the experiment to homogenize the samples that would otherwise show significant sedimentation over several days.

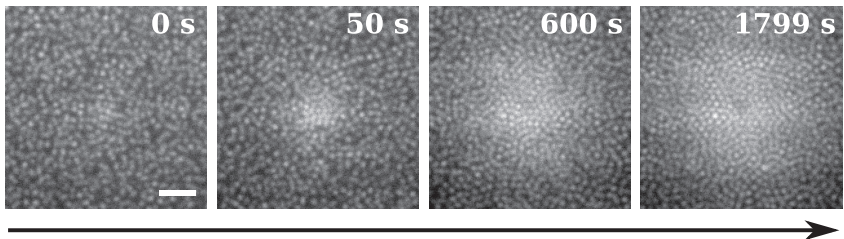

Time

FIG. 1. Confocal imaging snapshots showing the growth of a polycrystalline structure (particle radius $a=0.40 \mu \mathrm{m}$ ). At $t=0 \mathrm{~s}$, the laser power is switched from $0.2 \mathrm{~W}$ to $0.7 \mathrm{~W}$ in a sample of bulk volume fraction $\phi_{\infty}=0.25$ (scale bar equal to $5 \mu \mathrm{m}$ ). The heated titania particle at the center is barely visible as it is slightly out of the focal plane because of gravity.

Samples were placed on a setup consisting of an Olympus IX81 microscope with Olympus UPlanSApo $60 \times, 1.20$ numerical aperture water immersion objective. Observation of the PMMA particles was done by confocal microscopy using a 532-nm excitation laser (JUNO Nd:YVO4, $500 \mathrm{~mW}$ ), a Yokogawa CSU-X1 confocal unit, and an EMCCD Andor camera (iXon DV885KCS-VP).

Optical tweezing was obtained using a holographic optical tweezers (HOTkit, Arryx) setup combined to the microscope, although we did not use in this work the holographic features. In this configuration, the output of a 1064-nm-wavelength fiber laser (IPG, YLR-10-LP, $10 \mathrm{~W}$ ) passes successively through a half waveplate and a 50:50 beam-splitter cube, is reflected on a spatial light modulator (SLM) $(512 \times 512$, Boulder Nonlinear Systems) that is here turned off and thus essentially used as a mirror, and is directed back on the beam splitter, after which it passes through a telescope. In the holographic configuration, the telescope contains a mask at the focal point of the lenses to block the zeroth order of the beam diffracted by the SLM. This mask was removed here so that the main beam could be used for trapping. After the telescope, the beam has a Gaussian shape with a size slightly greater than the back aperture of the objective to which it is sent. The beam is directed to the back port of the microscope and reflects inside the microscope on a dichroic mirror (950-nm shortpass, Semrock FF01-950/SP) before finally entering the objective.

Before the start of an experiment, a titania particle was trapped close to the waist of the laser beam and the sample was moved to have the particle at about a 50- $\mu \mathrm{m}$ distance from the bottom and top walls of the cell. During this stage, a low laser power of $0.2 \mathrm{~W}$ was used. Powers indicate values from the laser controller. According to our estimate, for $1 \mathrm{~W}$ on the controller, the actual power passing through the sample was less than $235 \mathrm{~mW}$, as this value was measured at a location before entering the objective.

\section{RESULTS}

\section{A. Measurements of aggregate sizes}

When switching the laser power from a low value $(0.2 \mathrm{~W})$ to a higher value $P$ (up to $1.5 \mathrm{~W}$ ), aggregates start growing for sufficiently high $P$, until a steady state is reached. Figure 1 shows confocal images of the plane containing the laser focal point at different times for an experiment at the sample 
volume fraction $\phi_{\infty}=0.25, P=0.7 \mathrm{~W}$, and $a=0.4 \mu \mathrm{m}$. Movies S1-S3 in the Supplemental Material [33] also show the growth, accelerated 100 times, for $\phi_{\infty}=0.1$ and $P=$ $0.3,0.7$, and $1.1 \mathrm{~W}$, respectively. Clearly ordered and large polycrystalline structures are present for a long time, showing that a phase transition occurred. We can also see that the nearby fluid phase has a higher concentration of particles than the bulk of the fluid. In other samples with large PMMA particles of radius of $a=1.2 \mu \mathrm{m}$, we could identify the existence of face-centered-cubic (fcc) structures in the solid part of the aggregates. The aggregation does not occur when the laser is shined in the sample without a titania particle: For small particles of radius $a=0.15 \mu \mathrm{m}$, the laser is not able to trap the PMMA colloids, while for a radius of $1.2 \mu \mathrm{m}$, PMMA particles could be trapped occasionally because of an imperfect refractive-index match, but no other particles aggregated around it.

From movies consisting of time series of images as in Fig. 1, we have extracted the size of the solid condensed structures for colloids of radius $a=0.40 \mu \mathrm{m}$. Because of the small size of particles, the high density of the aggregates, and the limitation of the recordings to a two-dimensional plane, particle tracking could not be performed accurately. We instead measure a size based on a locally averaged quantity related to the mobility of the particles, which is described in Appendix A. We assume solid clusters have a spherical shape and thus characterize the size simply by the cluster radius $r_{\mathrm{i}}$. Figure 2(a) shows growth profiles for the bulk volume fraction $\phi_{\infty}=0.25$ when setting at $t=0 \mathrm{~s}$ the laser power from $0.2 \mathrm{~W}$ (for which there are no aggregates) to various values of $P$. The size increases faster with higher laser power. It also reaches higher steady-state values, as can be seen at least on the low $P$ curves. This size increase with time is well fitted empirically to exponential approaches to a finite radius $r_{\mathrm{i}}(t)=r_{\infty}+\left(r_{0}-r_{\infty}\right) e^{-t / \tau}$, thus giving estimates of the final size $r_{\infty}$ of the aggregates and the characteristic timescale of the growth of $\tau$. These two quantities are shown in Figs. 2(b) and 2(c) as functions of $\phi_{\infty}$ and $P$. In Fig. 2(b), the final size grows linearly with laser power and the dependence on the volume fraction is superlinear, as is evidenced by the linear fit for each volume fraction whose slope is plotted in the inset. The timescale of growth $\tau$ shows very scattered data with no clear dependence on either $P$ or $\phi_{\infty}$.

\section{B. Origin of the formation of aggregates}

In the experiments, aggregates formed only in the presence of the trapped central oxide particle. Their size can be much greater than the spatial window in which optical forces could act on the surrounding colloid particles, since the spot size estimated from the Rayleigh criterion is $1.2 \lambda / N A \approx 1 \mu \mathrm{m}$, assuming a uniformly lit objective. Hence, we can rule out the possibility that the aggregation emerges from optical forces on the colloidal particles. Instead, we show that the particles are subject to thermophoresis, due to the heating of the trapped titania particle by the tweezers laser.

We have tested that PMMA particles move towards hot regions in a temperature gradient, that is, by thermophoresis with a negative Soret coefficient. In a sample of PMMA colloids (without titania particles) containing a platinum wire
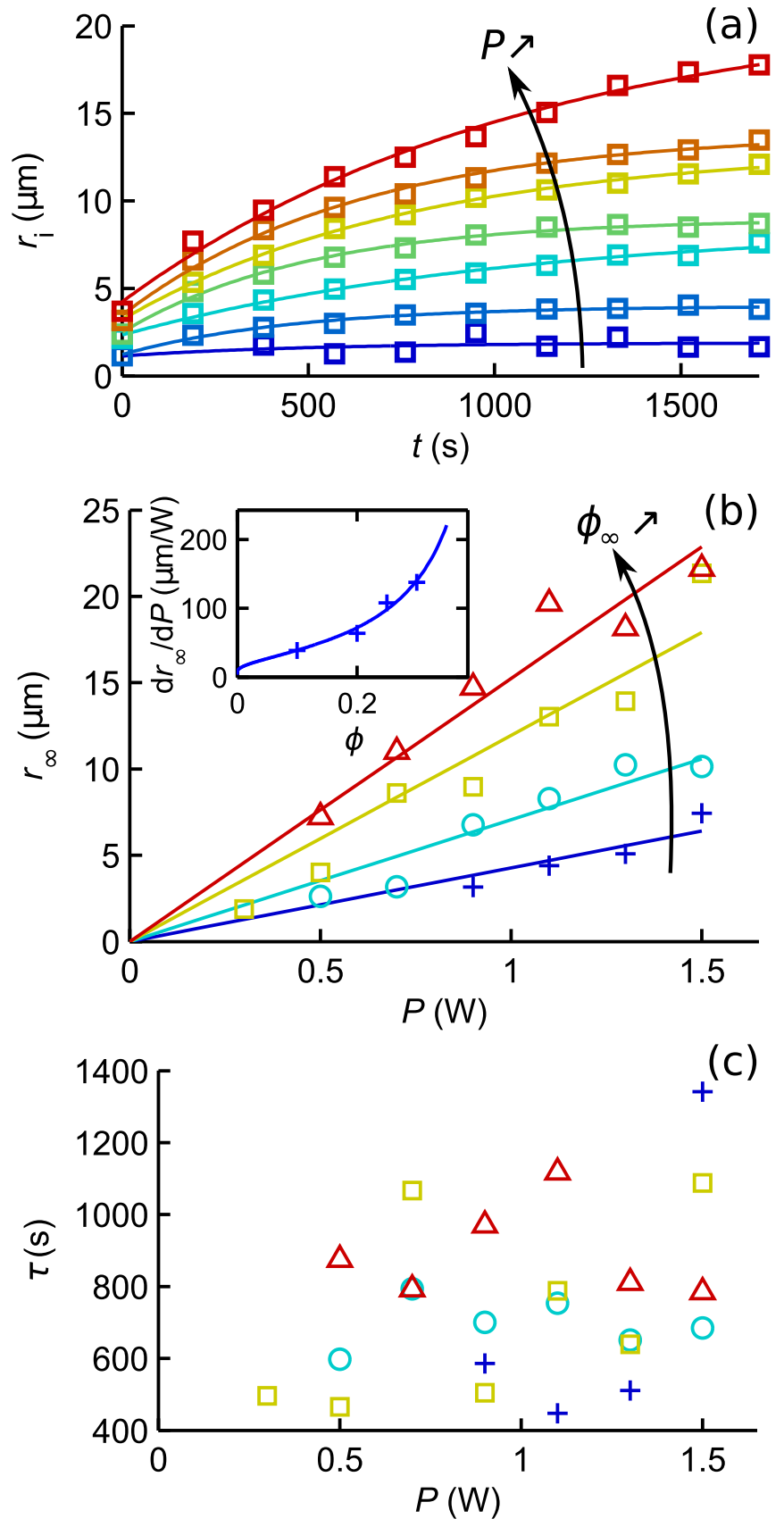

FIG. 2. Size measurements of the condensed part of the aggregates for PMMA colloids of radius $a=0.40 \mu \mathrm{m}$. (a) Size growth as a function of time for $\phi_{\infty}=0.25$ and $P=0.3,0.5,0.7,0.9,1.1$, 1.3 , and $1.5 \mathrm{~W}$ (from blue to red). The experiments (markers) are well fitted by exponential convergences (lines). (b) Final size $r_{\infty}$ of the polycrystalline clusters depending on laser power and volume fraction [markers show $\phi_{\infty}=0.1(+), 0.2(\circ), 0.25(\square)$, and $0.3(\triangle)$ ]. For each volume fraction, the lines represent proportional fits. The slope of these fits is shown in the inset, depending on the volume fraction (markers). The data are fitted to the model described by Eq. (4) (line). (c) Characteristic growth time $\tau$ depending on laser power and volume fraction [same coloring as in (b)].

of diameter $60 \mu \mathrm{m}$, we flow an electric current through the wire to heat the fluid locally and create a temperature gradient. Confocal movies imaging planes cutting through a diameter of the wire (movie S4 in [33]) and a cross section (movie S5 in 
[33]) were taken. Shortly after the start of the movies that are accelerated 20 times, a current of $50 \mathrm{~mA}$ is applied to the wire, which is turned off after several minutes. The times at which the current is turned on and off are evidenced by the wire moving to the left or right because of dilation. These movies show that particles aggregate around the wire and that no convective flows arise (movie S4 in [33], the gravitation field is vertical). Moreover, cutting the current leads to the aggregates disappearing within a few minutes. These tests confirm that the aggregation originates from thermophoresis and not from optical forces in the experiments with the trapped titania particles. They also rule out any photoinduced phenomenon as seen by Kim et al. [34], which is further supported by the fact that we do not see any strong dependence of $\tau$ on $\phi_{\infty}$ as opposed to their experiments.

\section{Model for the steady-state size}

To support further that the thermal gradient is the cause of the aggregation, we show below that the experiments in Fig. 2 are consistent with a model based on thermophoresis. We assume that significantly more laser light is absorbed by the titania particle than the fluid or the PMMA particles, as we see no aggregates when no titania particle is trapped. Therefore, the central metal-oxide particle acts as a small pointlike heat source that generates a temperature gradient with good spherical symmetry. We assume that the oxide particle is heated to a surface temperature $T_{0}$ that is proportional to the laser power $T_{0}=\alpha P$, which is true if no nonlinear light-matter interactions occur in the particle. The particle creates in the fluid a localized temperature gradient that stabilizes quickly compared to the timescale of aggregates growth. Thus, we may assume a steady state that obeys the time-independent heat equation $\nabla^{2} T=0$, from which we obtain the spatial profile of the temperature field $T(r)$, where $r$ is the distance from the center of the oxide particle,

$$
T(r)=T_{\infty}+\frac{R_{0}}{r} \Delta T,
$$

where $\Delta T=T_{0}-T_{\infty}$, with $T_{\infty}$ the bath temperature far from the titania particle. PMMA particles in this gradient experience directed motion. Their flux $\mathbf{j}(r)$ through a shell at a position $r$ is

$$
\mathbf{j}(r)=-D(\phi) \nabla \phi \hat{\mathbf{e}}_{r}-D(\phi) S_{\mathrm{T}} \phi \nabla T \hat{\mathbf{e}}_{r},
$$

where $D(\phi)$ is the diffusion coefficient at $\phi, S_{\mathrm{T}}$ the Soret coefficient, and $\hat{\mathbf{e}}_{r}$ the unit vector normal to the shell surface. In this equation, the first term corresponds to thermal diffusion and the second to the thermophoretic flux. Solving the equation $\mathbf{j}=0$ gives the steady-state concentration profile, valid in the fluid phase

$$
\phi(r)=\phi_{\infty} \exp \left(\frac{R}{r}\right),
$$

with $R=-S_{\mathrm{T}} \Delta T R_{0}$, by imposing the boundary condition $\phi=\phi_{\infty}$ at $r \rightarrow \infty$. If there is no metastability in the system, the density of the fluid phase near the fluid-solid interface should always be close to the same value, the volume fraction of the phase transition $\phi_{\mathrm{t}}$. Therefore, in the steady state, the position of the interface can be obtained using a second boundary condition $\phi\left(r_{\infty}\right)=\phi_{\mathrm{t}}$, where $r_{\infty}$ is the position of the interface in the steady state. We deduce from this condition that the position of the interface follows

$$
r_{\infty}\left(\phi_{\infty}, P\right)=\frac{-S_{\mathrm{T}} \alpha R_{0} P}{\ln \frac{\phi_{\mathrm{t}}}{\phi_{\infty}}} .
$$

This equation agrees with the linear dependence of $r_{\infty}$ on $P$ and the nonlinearity with $\phi_{\infty}$ observed in the experiments. It shows that the size diverges when $\phi_{\infty} \rightarrow \phi_{t}$, as minute concentration changes would then lead to the phase transition. It can be noted that the only input from thermodynamics in determining $r_{\infty}$ is the value of $\phi_{\mathrm{t}}$. We fitted the parameters $-S_{\mathrm{T}} \alpha R_{0}$ and $\phi_{\mathrm{t}}$ to the data in the inset of Fig. 2(b) using Eq. (4) and obtained $-S_{\mathrm{T}} \alpha R_{0}=6.57 \mu \mathrm{m} \mathrm{W}^{-1}$ and $\phi_{\mathrm{t}}=$ 0.458 . The fit (solid line) shows excellent agreement with the experiments. The value found for $\phi_{t}$ is very close to the freezing point of hard spheres at 0.494 , which is expected in this system where the charges of the colloids are screened [9,13-15]. To determine $S_{\mathrm{T}}$, an estimate of $\Delta T$ is required. Temperatures at such small scales are hard to measure accurately. A rough estimate was obtained however by using a sample of titania particles in a lutidine-water mixture $(29.13 \%$ in mass) that demixes at a temperature of $33.7^{\circ} \mathrm{C}$ [35]. By controlling the external temperature and measuring the laser power from which demixing starts when trapping a titania particle, we were able to estimate the coefficient $\alpha$ to $\alpha \sim$ $4 \mathrm{~K} \mathrm{~W}^{-1}$, from which we deduced $S_{\mathrm{T}} \sim-1 \mathrm{~K}^{-1}$ for particles with a $0.40-\mu \mathrm{m}$ radius. For particles with a radius of $1.2 \mu \mathrm{m}$, on the other hand, it was harder to obtain reliable exponential convergence fits as in Fig. 2(a): At long times, the size tended to fluctuate around an average value, because the reorganization of internal structures occurred. Movie S6 in [33] and Fig. 6 show the aggregate size growth in a case where these fluctuations were small, with $\phi_{\infty}=0.10$ and $P=1.5 \mathrm{~W}$. We found here $r_{\infty}=17 \mu \mathrm{m}$ and $\tau=680 \mathrm{~s}$ (see Appendix B). Compared to the value of $r_{\infty}=6 \mu \mathrm{m}$ of the linear fit in the same conditions in Fig. 2(b), for which particles were three times smaller, we found that $S_{\mathrm{T}}$ is proportional to $a$. This is consistent with a size-independent thermophoretic mobility [32,36] $D_{\mathrm{T}}=D S_{\mathrm{T}}$, assuming that $D(\phi) \approx k_{\mathrm{B}} T / 6 \pi \eta a$, with $k_{\mathrm{B}}$ the Boltzmann constant and $\eta$ the solvent viscosity.

In the model above, we assumed that the thermal conductivity of the mixture was independent of the concentration of PMMA particles. Since these have a higher conductivity than the solvent, their concentration around the central colloid changes the temperature gradient. We briefly present here how this changes the equations governing the steady-state distribution of the particles. Full justification of the approximations and details on the calculation are provided in Appendix C. Since the thermal conductivities of the particles $k_{\mathrm{p}}$ and of the solvent $k_{\text {sol }}$ only differ moderately, it is appropriate to model the overall thermal conductivity $k$ of the suspension as the linear combination $k=\phi k_{\mathrm{p}}+(1-\phi) k_{\text {sol }}$ (see Appendix C). This makes $\nabla T$ dependent on the $\phi(r)$ distribution in Eq. (2). As it is shown in Appendix $C$, this changes the differential equation satisfied by $\phi(r)$ and its solution, when applying appropriate boundary conditions. Instead of Eq. (3) we obtain

$$
\phi(r)=\frac{1}{\tilde{k}} W_{0}\left(\tilde{k} \phi_{\infty} e^{\tilde{k} \phi_{\infty}+R / r}\right),
$$


where $\tilde{k}=k_{\mathrm{p}} / k_{\mathrm{sol}}-1>0$ and $W_{0}$ is the principal branch of the Lambert function. The position of the interface in the steady state $r_{\infty}$ then becomes

$$
r_{\infty}\left(\phi_{\infty}, P\right)=\frac{-S_{\mathrm{T}} R_{0} \alpha P}{\ln \frac{\phi_{\mathrm{t}}}{\phi_{\infty}}+\tilde{k}\left(\phi_{t}-\phi_{\infty}\right)},
$$

instead of Eq. (4). While the difference in thermal conductivities is not negligible, using this more accurate model to fit $-S_{\mathrm{T}} \alpha R_{0}$ and $\phi_{\mathrm{t}}$ gives very similar values (see Appendix $\mathrm{C}$ for a comparison of the two models).

\section{Dynamics of growth}

Understanding the dynamics of the growth of aggregates is more complex than determining their final sizes. For laser powers sufficiently high to form clusters, the size starts to increase as soon as the power is raised. There is therefore no sign of existence of critical nuclei and the potential gradient is, in that case, strong enough in the vicinity of the central particle to overcome the energy barrier for nucleation. Using slightly lower laser powers to try to go below this barrier lead to no clustering at all. These two observations suggest that critical nuclei must have a size smaller than the typical range of the decay of the potential, i.e., smaller than $R_{0}$ [see Eq. (1)]. Even with a negligible effect of nucleation, the characteristic time $\tau$ is difficult to obtain analytically as solving the concentration variation $\partial \phi / \partial t+\nabla \cdot \mathbf{j}=0$ requires the inclusion of the advancement of the interface over time and how the flux is written at this location.

Concerning the advancement, it may be limited by either the reaction rate to grow crystallites in a supersaturated layer or the supply of PMMA colloids from thermophoresis and diffusion [37]. We noted that in the fluid phase close to the interface, the concentration is high only in a layer of a few colloid diameters. Therefore, we can rule out that we are in a reaction-rate-limited regime, as there is no accumulation of dense fluid. In a diffusion-limited scenario without thermophoresis, a depletion of particles in the fluid near the growing crystallites is expected [37]. However, we did not observe any depletion. Furthermore, Fig. 2(a) shows higher growth rates in short times for larger temperature gradients. Hence, we could refer to the phenomenon as thermophoresis limited rather than diffusion limited. This denomination should however be taken with care, as there is a complex coupling between diffusion and thermophoresis in setting the concentration field. This illustrates that, in out-of-equilibrium systems, the dynamics of phase transitions cannot be simply classified as either reaction-rate or diffusion limited.

\section{E. Internal structure of aggregates}

While we have mainly studied a case where we obtained polycrystalline structures, Fig. 3 shows, using $1.2-\mu$ m-radius colloids, that different types of clusters can be obtained and we have seen amorphous states [Fig. 3(a)], crystalline states [Fig. 3(b)], and structures with icosahedral order [Fig. 3(c)]. We do not have a full quantitative characterization of these systems, but we may explain these phenomena on a qualitative level. First, frustration against crystallization can emerge from various sources [38]. The image in Fig. 3(a) shows that, in
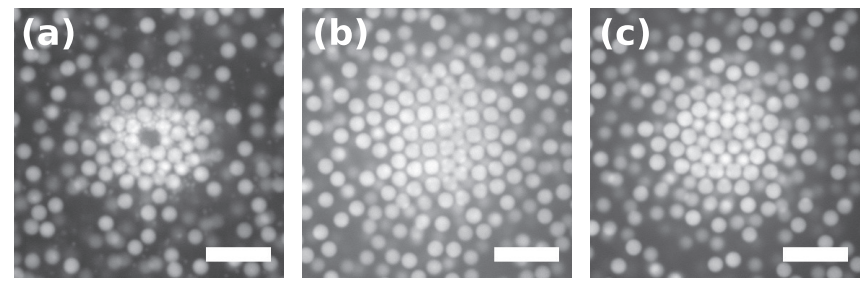

FIG. 3. Internal structure of condensed clusters (colloid radius equal to $1.2 \mu \mathrm{m}$ and scale bar equal to $10 \mu \mathrm{m}$ ). Depending on the experimental conditions, (a) amorphous, (b) polycrystalline with at least fcc crystallites, and (c) structures with icosahedral order can be obtained. In (b) and (c) the colloid solution was filtered to remove particles much smaller than the $1.2-\mu \mathrm{m}$-radius particles that are visible in (a). The high polydispersity in (a) is likely to have prevented crystallization. Furthermore, the density match was better in (b) (21.6\% cis-decalin/bromocyclohexane) than in (a) and (c) $(20 \%)$, which can affect the kinetics of particles in addition to the clusters.

that experiment, very small particles were also present, so this polydispersity of the system may be the reason for the amorphous formation. Even though the overall size polydispersity is small, there is a possibility that a small portion of particles with very different sizes may have a significant impact on the ordering. Next, the formation of clusters with icosahedral symmetry like in Fig. 3(c) may emerge from self-induced spherical confinement. It was shown [39] that hard spheres compressed under spherical confinement spontaneously crystallize into icosahedral clusters that are entropically favored over the bulk fcc crystal structure. In our experiments, confinement intervenes in two ways that are somewhat different from Ref. [39], but still with spherical symmetry: through the temperature gradient that induces a soft and long-range component and through the central spherical impurity that hinders PMMA particles from reaching positions of radius lower than $R_{0}+a$ and that represents an "inverse spherical confinement" with a forbidden void. Why no long-range icosahedral order exists in Fig. 3(b) might then be due to different kinetics of particle addition to the cluster, or to slightly different interparticle interactions as the density and refractive index match was more accurate in Fig. 3(b) than in Fig. 3(c). Since the first layers of the icosahedral structure form very early and tend to remain stable as an aggregate grows (see movie S7 in [33], 100 $\times$ play speed), the symmetry of particle arrangement initially formed around the titanium particle is likely to have a substantial impact on the later ordering. These examples show very rich possibilities of ordering behaviors in localized inhomogeneous fields. We expect that, by manipulating the shape of the temperature gradient and/or the steric constraints due to the metal-oxide particle by changing its shape, the symmetry selection of the clusters of particles should be affected [40].

\section{CONCLUSION}

To summarize, we studied how colloids undergo phase transitions or transitions to various states under a temperature gradient formed by a pointlike heat source. We found that thermophoresis leads to the densification of colloids around 
the point, resulting in local crystallization, glass transition, and the formation of structures with icosahedral order that could be a precursor to a quasicrystal.

This setup provides the advantage of exploring the state growth in unconventional potentials that have sharp variations in the range of a few particle diameters but that are still smoother than hard-wall boundary conditions. Fluctuations in the shape of the interface from a perfect sphere may, for example, be affected by this. The unique spatial profile of the potential introduces not only the radial symmetry but also strong spatial inhomogeneity to the externally induced interparticle interaction. Moreover, growth can be observed by starting from highly nonequilibrium conditions, and our study indicates that this leads to structures built layer by layer (although we occasionally saw particle rearrangements in clusters). This is in stark contrast with the typical first-order transition in a thermal system, which takes place by overcoming the free-energy barrier. Finally, the central particle acting as a heat source can act as a source of geometrical frustration, depending upon its size and shape. These, together with the various structures we could obtain, suggest a possible role of the structure of the initially formed particle layer and hence the size and shape of the central impurity on the determination of the ordered structures, which could be studied further.

\section{ACKNOWLEDGMENTS}

We thank Isaac Theurkauff for his help on the optical tweezers setup and Taiki Yanagishima for his knowledge on colloids synthesis as well as useful discussions with Yoav Tsori. We thank the Institut de Biologie de Valrose and the PRISM platform for the use of a confocal microscope to test the samples with a heated platinum wire. This study was partially supported by Grants-in-Aid for Specially Promoted Research (Grant No. JP25000002) and Scientific Research (A) (JP18H03675) from the Japan Society for the Promotion of Science (JSPS). N.B. is grateful to JSPS for a JSPS Postdoctoral Fellowship (JP15F15769).

\section{APPENDIX A: MEASUREMENT OF THE SIZE OF AGGREGATES}

Here we explain how to determine the size of the solid part of the aggregates from movies consisting of time series of images taken at 1-s intervals. Because of the small size of the PMMA particles, the high density of the aggregates, and the limitation of the recordings to a two-dimensional plane, particle tracking could not be performed accurately. We instead measure a locally averaged quantity related to the mobility of the particles. The steps are illustrated in Figs. 4 and 5 .

After applying a bandpass Fourier filter to the images [Fig. 4(a)], we measure the subtraction of frames separated by a time delta of $10 \mathrm{~s}$ [Fig. 4(b)], a duration during which free particles typically diffuse by more than one diameter $\left(\sqrt{2 D \Delta t}=2.0 \mu \mathrm{m}\right.$, with $D=k_{B} T / 6 \pi \eta a$, setting $\eta=$ $2.6 \mathrm{mPas})$. The frame difference maps are then split into square areas called blocks, in a grid with the size of each block set to 9 pixels $(1.0 \mu \mathrm{m})$, of the order of a PMMA particle diameter. For each block, we then calculate the standard deviation of the signal within the block and normalize it by the average intensity (averaged over the block pixels and the two frames considered) [Fig. 4(c)]. The map in Fig. 4(c) shows a well-defined central region with very small motion of the colloids whose size matches the size of the ordered part of the aggregate seen in Figs. 4(a) and 4(b). Since a block can typically contain at most one particle, the block signal can still fluctuate significantly for colloids in the gas phase, especially for low volume fractions. Hence, a further average is performed by calculating and averaging normalized block standard deviation maps at different times $t, t+\Delta t, \ldots, t+$ $n_{\text {avg }} \Delta t$, with $n_{\text {avg }}=8$. Such an averaged map is displayed in
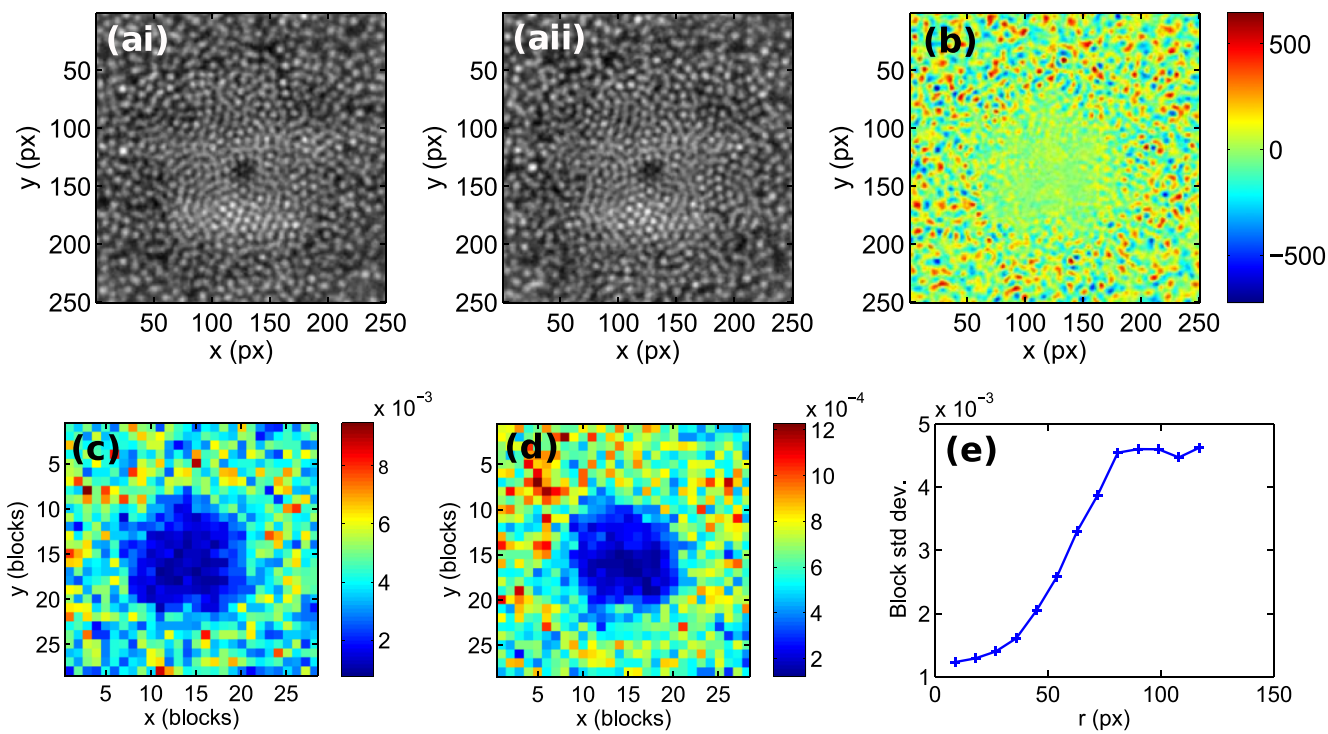

FIG. 4. Extracting a local quantity related to the mobility of the particles. (a) Two frames (Fourier filtered) and separated by $10 \mathrm{~s}$. (b) Intensity difference between the two frames in (a). (c) Block standard deviation map. (d) Block standard deviation map averaged over several time windows. (e) Angle-averaged radial variation of the block standard deviation. 


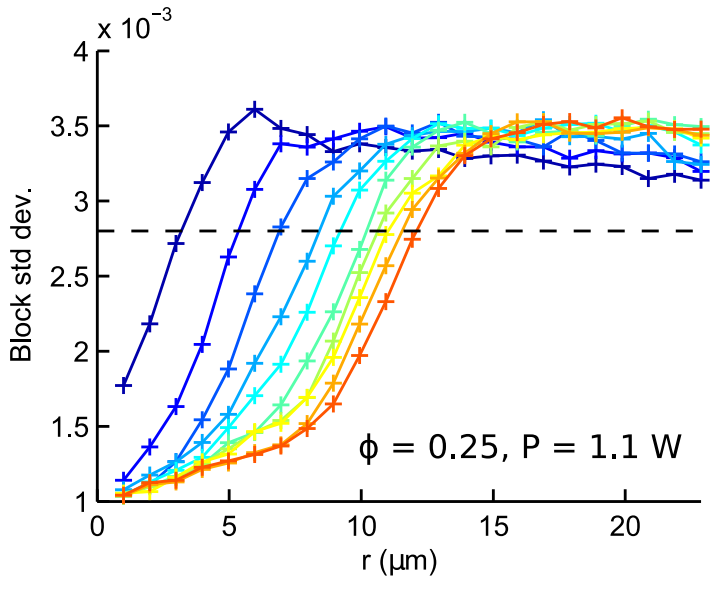

FIG. 5. Radial evolution of the normalized block standard deviation. The curves represent different times (from blue to red, 0-1710 s in 190-s steps) and show a sharp increase as the solid-fluid interface is passed. Applying a threshold (dashed line) gives an estimate of the position of the interface.

Fig. 4(d). To extract an aggregate size, we reduce the map to a signal depending on the distance $r$ to the center of the trapped titania particle, averaging over all angles [Fig. 4(e)]. This signal shows a sharp increase in the mobility of the particles when traversing the interface between the two phases. Finally, we use a threshold of 2.8 to obtain the aggregate size. The value was chosen to match interface positions determined manually on a random set of movies.

An advantage of this method is that regions at different concentrations, but with particles moving freely, lead to the same value of the block standard deviation. The main caveat is that while the block standard deviation value decreases monotonically with increasing confinement, there is a nonlinearity that arises from the intensity profile of a single particle as it affects how the difference in the map of frames evolves with the distance a particle has moved. This nonlinearity is not meaningful as it has no relation to the fluctuations of the particle positions.

\section{APPENDIX B: AGGREGATE SIZE FOR PARTICLES OF 1.2- $\mu \mathrm{m}$ RADIUS}

Movie S6 (100× play speed) in [33] shows the growth of an aggregate of PMMA particles of radius $a=1.2 \mu \mathrm{m}$ and for $\phi_{\infty}=0.10$ and $P=1.5 \mathrm{~W}$. While most of our experiments with these particles showed strong fluctuations of the size around a final value (after the initial growth), this particular test has limited fluctuations at long times, as can be seen in Fig. 6. Fitting a characteristic final size and time of growth gives $r_{\infty}=17 \mu \mathrm{m}$ and $\tau=680 \mathrm{~s}$.

\section{APPENDIX C: MODEL WITH CONCENTRATION-DEPENDENT THERMAL CONDUCTIVITY}

In our system, solvent and PMMA particles have different thermal conductivities. This should modify the temperature gradient, depending on the concentration field, in a complex manner due to their coupling. We present here a model that

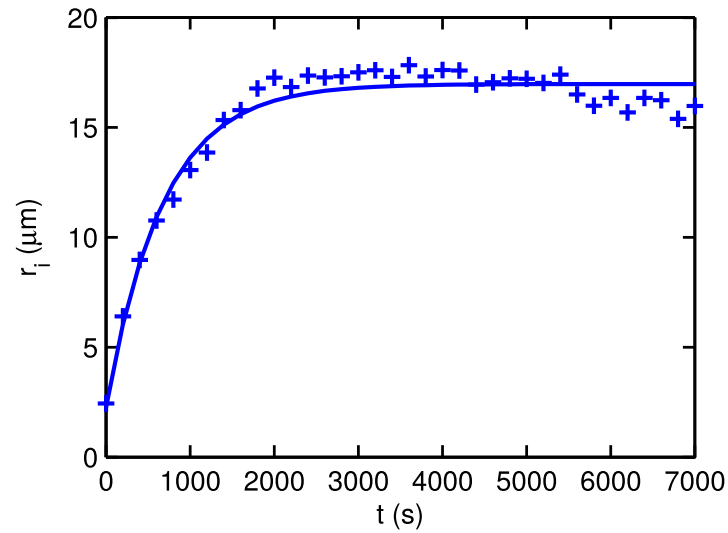

FIG. 6. Aggregate size $r_{\mathrm{i}}$ for $a=1.2 \mu \mathrm{m}, \phi_{\infty}=0.10$, and $P=1.5 \mathrm{~W}$ (markers show the experiment and the solid line the fit).

uses a simple relation between thermal conductivity and concentration and derives formulas for the concentration field and the size of the aggregates.

The Maxwell mean-field theory, initially applied to electric transport [41], is commonly used to estimate the thermal conductivity of colloidal suspensions, especially for nanoparticles. The theory provides formulas for the overall thermal conductivity of the suspension in two limit cases called upper and lower limits that correspond to different orderings of the particles. Most experimental data lie between the two limits [42]. These are

$$
\frac{k}{k_{\mathrm{sol}}}=1+\frac{3 \phi\left(k_{\mathrm{p}}-k_{\mathrm{sol}}\right)}{3 k_{\mathrm{sol}}+(1-\phi)\left(k_{\mathrm{p}}-k_{\mathrm{sol}}\right)}
$$

for the lower limit and

$$
\frac{k}{k_{\mathrm{sol}}}=\frac{k_{\mathrm{p}}}{k_{\mathrm{sol}}}\left[1-\frac{3(1-\phi)\left(k_{\mathrm{p}}-k_{\mathrm{sol}}\right)}{3 k_{\mathrm{p}}-\phi\left(k_{\mathrm{p}}-k_{\mathrm{sol}}\right)}\right]
$$

for the upper limit with $k, k_{\mathrm{p}}$, and $k_{\mathrm{sol}}$ the thermal conductivities of the suspension, the PMMA particles, and the solvent, respectively. Typical values for our system are $k_{\mathrm{p}}=$ 0.19 [43] and $k_{\mathrm{sol}}=0.12 \mathrm{~W} / \mathrm{m} / \mathrm{K}$ [44]. Figure 7 shows that

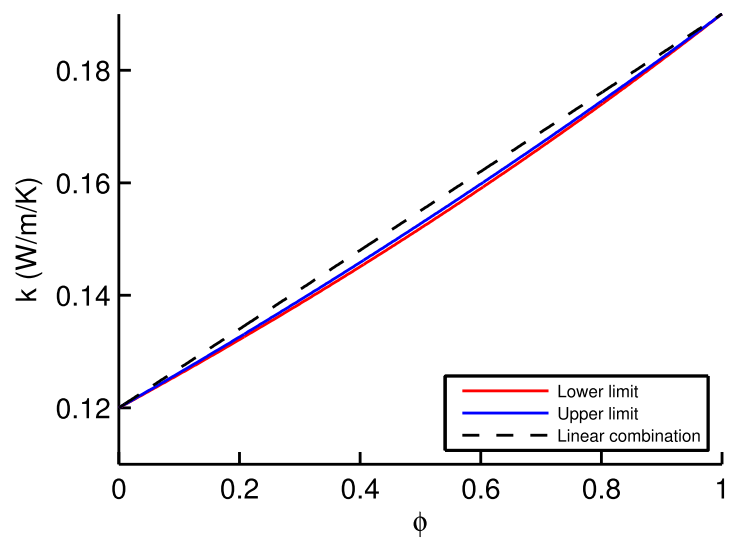

FIG. 7. Thermal conductivity of a colloidal suspension, according to the Maxwell mean-field theory. For PMMA particles density matched in a cis-decalin/bromocyclohexane mixture, the two limits of the theory are, for all volume fractions, close to a simple linear combination of the conductivities of the colloids and the solvent. 
for this moderate discrepancy between the two thermal conductivities, the overall conductivity of the suspension given by the Maxwell theory can be approximated by the linear combination

$$
k=\phi k_{\mathrm{p}}+(1-\phi) k_{\mathrm{sol}} \text {. }
$$

To simplify the calculation below, we will therefore model the conductivity in our system with Eq. (C3).

We denote by $P_{\mathrm{h}}$ the power of heat dissipated by the central colloid. In the steady state, the heat flow through any sphere at radius $r>R_{0}$ should equal $P_{\mathrm{h}}$. In a spherically symmetric configuration, the heat flux is $\mathbf{q}=-k \frac{d T}{d r} \hat{\mathbf{e}}_{r}$, so

$$
\frac{P_{\mathrm{h}}}{\pi r^{2}}=-k \frac{d T}{d r},
$$

where $k$ now depends on the concentration of colloids according to Eq. (C3). As in the main text, the motion of the PMMA particles is governed in the steady state by the balance of flux due to diffusion and the thermophoretic flux that are both in the $\hat{\mathbf{e}}_{r}$ direction:

$$
0=-D \frac{d \phi}{d r}-D S_{\mathrm{T}} \phi \frac{d T}{d r} .
$$

If there is a phase transition with a solid aggregate for $r<r_{\infty}$, this equation is then only valid for $r>r_{\infty}$. In this range, Eqs. (C3)-(C5) give the differential equation in $\phi(r)$,

$$
r^{2}(1+\tilde{k} \phi) \frac{d \phi}{d r}-\frac{S_{\mathrm{T}} P_{\mathrm{h}}}{\pi k_{\mathrm{sol}}} \phi=0,
$$

where $\tilde{k}=k_{\mathrm{p}} / k_{\mathrm{sol}}-1$. It is appropriate to express $P_{\mathrm{h}}$ as a function of quantities measured experimentally. Since $P_{\mathrm{h}}$ is not dependent on how the heat propagates in the fluid, we obtain it in our work from the measurement (see the main text) of the temperature difference between the surface of the colloids and the temperature far from the center, $\Delta T=$ $T_{0}-T_{\infty}$, in a fluid without colloids. Then $P_{\mathrm{h}}$ is deduced by integrating $\frac{P_{\mathrm{h}}}{\pi r^{2}}=-k_{\mathrm{sol}} \frac{d T}{d r}$ between $R_{0}$ and $r \rightarrow \infty$. We obtain

$$
P_{\mathrm{h}}=\pi k_{\mathrm{sol}} R_{0} \Delta T
$$

and Eq. (C6) becomes

$$
r^{2}(1+\tilde{k} \phi) \frac{d \phi}{d r}+R \phi=0,
$$

with $R=-S_{\mathrm{T}} \Delta T R_{0}$. The solutions to this equation are expressed in terms of the Lambert $W$ function, which only has two branches in real space. Imposing the constraint that $\tilde{k}>0$, the relevant branch is the principal branch $W_{0}$ and we deduce

$$
\phi(r)=\frac{1}{\tilde{k}} W_{0}\left(\tilde{k} e^{C+R / r}\right),
$$

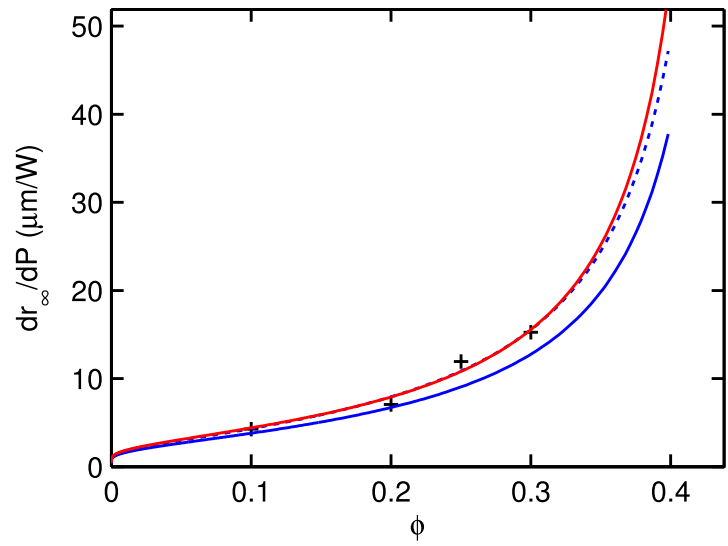

FIG. 8. Comparison of the estimates of aggregate sizes using the models with constant thermal conductivity [Eq. (4)] (dashed blue curve) (the same as in the main text) and $\phi$-dependent conductivity [Eq. (C11)] (solid blue curve) with the same values of the fitting parameters $\phi_{\infty}$ and $-S_{\mathrm{T}} R_{0} \alpha$. Then the red solid curve is the fit of $\phi$-dependent conductivity (C11) with different parameters.

with $C$ a constant. The constant is obtained by applying the boundary condition that the concentration tends to $\phi_{\infty}$ when $r \rightarrow \infty$. Using the property that $z=W_{0}\left(z e^{z}\right)$ for any $z$ value, we get $C=\ln \phi_{\infty}+\tilde{k} \phi_{\infty}$ and the concentration field

$$
\phi(r)=\frac{1}{\tilde{k}} W_{0}\left(\tilde{k} \phi_{\infty} e^{\tilde{k} \phi_{\infty}+R / r}\right) .
$$

For $\tilde{k} \rightarrow 0$, we recover the concentration field for constant thermal conductivity [Eq. (3)].

Following the same reasoning as in the case of constant thermal conductivity and setting $r$ to the steady-state interface position $r_{\infty}$, for which $\phi\left(r_{\infty}\right)=\phi_{\mathrm{t}}$, we find the position of the interface at

$$
r_{\infty}=\frac{-S_{\mathrm{T}} R_{0} \alpha P}{\ln \frac{\phi_{\mathrm{t}}}{\phi_{\infty}}+\tilde{k}\left(\phi_{t}-\phi_{\infty}\right)} .
$$

Figure 8 compares this model (solid blue line) with variable thermal conductivity to the simpler model in the main text (dashed line), taking for $-S_{\mathrm{T}} R_{0} \alpha$ and $\phi_{t}$ the values obtained from fitting the simplified model. The two models only match at low volume fractions and differ by a factor $1+\tilde{k} \phi_{t}$ when approaching $\phi_{t}$. In the moderate range of volume fractions we have explored (up to $\phi_{\infty}=30 \%$ ), the maximum discrepancy between the two models is about $20 \%$. Fitting the experiments to Eq. (C11) (red curve) gives similar parameters as for the model with constant conductivity: $-S_{\mathrm{T}} R_{0} \alpha=7.47 \mu \mathrm{m} / \mathrm{W}$ and $\phi_{t}=0.445$. The discrepancy between the two models appears to affect mainly the $-S_{\mathrm{T}} R_{0} \alpha$ parameter.
[1] H. Löwen, Eur. Phys. J. Spec. Top. 222, 2727 (2013).

[2] U. Gasser, Science 292, 258 (2001).

[3] P. Tan, N. Xu, and L. Xu, Nat. Phys. 10, 73 (2013).

[4] A. Kasper, E. Bartsch, and H. Sillescu, Langmuir 14, 5004 (1998).

[5] W. K. Kegel and A. van Blaaderen, Science 287, 290 (2000).
[6] E. R. Weeks, J. C. Crocker, A. C. Levitt, A. Schofield, and D. A. Weitz, Science 287, 627 (2000).

[7] E. R. Weeks and D. A. Weitz, Phys. Rev. Lett. 89, 095704 (2002).

[8] P. Schall, D. A. Weitz, and F. Spaepen, Science 318, 1895 (2007). 
[9] M. Leocmach and H. Tanaka, Soft Matter 9, 1447 (2013).

[10] J. E. Hallett, F. Turci, and C. P. Royall, Nat. Commun. 9, 3272 (2018).

[11] R. Pinchaipat, M. Campo, F. Turci, J. E. Hallett, T. Speck, and C. P. Royall, Phys. Rev. Lett. 119, 028004 (2017).

[12] P. J. Lu, E. Zaccarelli, F. Ciulla, A. B. Schofield, F. Sciortino, and D. A. Weitz, Nature (London) 453, 499 (2008).

[13] C. P. Royall, W. C. K. Poon, and E. R. Weeks, Soft Matter 9, 17 (2013).

[14] S. Arai and H. Tanaka, Nat. Phys. 13, 503 (2017).

[15] H. Tsurusawa, M. Leocmach, J. Russo, and H. Tanaka, Sci. Adv. 5, eaav6090 (2019).

[16] K. Loudiyi and B. J. Ackerson, Physica A 184, 1 (1992).

[17] Q.-H. Wei, C. Bechinger, D. Rudhardt, and P. Leiderer, Phys. Rev. Lett. 81, 2606 (1998).

[18] A. A. Verhoeff, F. A. Lavergne, D. Bartolo, D. G. A. L. Aarts, and R. P. A. Dullens, Soft Matter 11, 3100 (2015).

[19] K. Zahn, R. Lenke, and G. Maret, Phys. Rev. Lett. 82, 2721 (1999).

[20] J. Zhao, P. Papadopoulos, M. Roth, C. Dobbrow, E. Roeben, A. Schmidt, H.-J. Butt, G. K. Auernhammer, and D. Vollmer, Eur. Phys. J. Spec. Top. 222, 2881 (2013).

[21] A. van Blaaderen, M. Dijkstra, R. van Roij, A. Imhof, M. Kamp, B. W. Kwaadgras, T. Vissers, and B. Liu, Eur. Phys. J. Spec. Top. 222, 2895 (2013).

[22] K.-Q. Zhang and X. Y. Liu, Nature (London) 429, 739 (2004).

[23] L. J. Moore, R. D. Dear, M. D. Summers, R. P. A. Dullens, and G. A. D. Ritchie, Nano Lett. 10, 4266 (2010).

[24] H.-R. Jiang, H. Wada, N. Yoshinaga, and M. Sano, Phys. Rev. Lett. 102, 208301 (2009).

[25] H.-D. Deng, G.-C. Li, H.-Y. Liu, Q.-F. Dai, L.-J. Wu, S. Lan, A. V. Gopal, V. A. Trofimov, and T. M. Lysak, Opt. Express 20, 9616 (2012).

[26] Y. Peng, F. Wang, Z. Wang, A. M. Alsayed, Z. Zhang, A. G. Yodh, and Y. Han, Nat. Mater. 14, 101 (2014).
[27] Z. Wang, F. Wang, Y. Peng, and Y. Han, Nat. Commun. 6, 6942 (2015).

[28] R. Piazza and A. Parola, J. Phys.: Condens. Matter 20, 153102 (2008).

[29] A. Würger, Rep. Prog. Phys. 73, 126601 (2010).

[30] R. Piazza, Soft Matter 4, 1740 (2008).

[31] S. Duhr and D. Braun, Appl. Phys. Lett. 86, 131921 (2005).

[32] A. Würger, Langmuir 25, 6696 (2009).

[33] See Supplemental Material at http://link.aps.org/supplemental/ 10.1103/PhysRevResearch.1.033200 for movies of aggregate growth and colloids moving around a heated platinum wire.

[34] Y. Kim, A. A. Shah, and M. J. Solomon, Nat. Commun. 5, 3676 (2014).

[35] S. Z. Mirzaev, R. Behrends, T. Heimburg, J. Haller, and U. Kaatze, J. Chem. Phys. 124, 144517 (2006).

[36] M. Braibanti, D. Vigolo, and R. Piazza, Phys. Rev. Lett. 100, 108303 (2008).

[37] U. Gasser, J. Phys.: Condens. Matter 21, 203101 (2009).

[38] H. Tanaka, Eur. Phys. J. E 35, 113 (2012).

[39] B. De Nijs, S. Dussi, F. Smallenburg, J. D. Meeldijk, D. J. Groenendijk, L. Filion, A. Imhof, A. Van Blaaderen, and M. Dijkstra, Nat. Mater. 14, 56 (2015).

[40] M. Hermes, E. C. M. Vermolen, M. E. Leunissen, D. L. J. Vossen, P. D. J. van Oostrum, M. Dijkstra, and A. van Blaaderen, Soft Matter 7, 4623 (2011).

[41] J. C. Maxwell, Treatise on Electricity and Magnetism (Clarendon, Oxford, 1873)

[42] S. Lotfizadeh, T. Desai, and T. Matsoukas, APL Mater. 2, 066102 (2014).

[43] M. J. Assael, S. Botsios, K. Gialou, and I. N. Metaxa, Int. J. Thermophys. 26, 1595 (2005).

[44] K. Youngri, Beyond equilibrium assemblies: Applying light, flow, and confinement, Ph.D. thesis, University of Michigan, 2016. 